\title{
Lymphopenia following pancreaticoduodenectomy is associated with pancreatic fistula formation
}

\author{
Joshua T. Cohen, Kevin P. Charpentier, Thomas J. Miner, William G. Cioffi, and Rachel E. Beard
}

Department of Surgery, Rhode Island Hospital, Warren Alpert Medical School of Brown University, Providence, RI, USA

\begin{abstract}
Backgrounds/Aims: Post-operative pancreatic fistulas (POPF) are a major source of morbidity following pancreaticoduodenectomy (PD). This study aims to investigate if persistent lymphopenia, a known marker of sepsis, can act as an additional marker of POPF with clinical implications that could help direct drain management. Methods: A retrospective chart review of all patients who underwent PD in a single hospital network from 2008 to 2018. Persistent lymphopenia was defined as lymphopenia beyond post-operative day \#3. Results: Of the 201 patients who underwent PD during the study period 161 patients had relevant laboratory data, 81 of whom had persistent lymphopenia. 17 patients with persistent lymphopenia went on to develop a POPF, compared to 7 patients without. Persistent lymphopenia had a negative predictive value of $91.3 \%$. Multivariate analysis revealed only persistent lymphopenia as being independently associated with POPF (HR 2.57, 95\% Cl 1.07-6.643, $p=0.039)$. Patients with persistent lymphopenia were more likely to have a complication requiring intervention $(56.8 \%$ vs $35.0 \%, p<0.001)$. Conclusions: Persistent lymphopenia is a readily available early marker of POPF that holds the potential to identify clinically relevant POPF in patients where no surgical drain is present, and to act as an adjunct of drain amylase helping to guide drain management. (Ann Hepatobiliary Pancreat Surg 2021;25:242-250)
\end{abstract}

Key Words: Lymphopenia; Post-operative pancreatic fistula; Pancreaticoduodenectomy

\section{INTRODUCTION}

Despite strategies to mitigate the sequelae of POPF following pancreaticoduodectomy, POPF continues to be a significant source of morbidity and mortality. Reported rates of POPF range from $3-45 \%$, with a $0-5 \%$ mortality rate. ${ }^{1-4}$ A systematic review of 60,000 patients demonstrated that $3.5 \%$ of all pancreaticoduodenectomy patients had a POPF that lead to organ failure, the need for re-operation, or death. An additional $8.9 \%$ of patients required antibiotic therapy or additional procedures such as percutaneous, endoscopic, or angiographic interventions as part of their POPF treatment. ${ }^{3}$ Management and prevention remains controversial with increasing attention dedicated to the need for prophylactic intraperitoneal drains at the time of the index operation and their optimal management in the postoperative period. ${ }^{5-8}$
Recent studies have offered conflicting data regarding the effect of drains on clinical outcomes after pancreaticoduodenectomy. Some studies suggest an increase in the number and severity of complications when drains are not left, whereas in others no differences were reported with or without drains. ${ }^{5,6}$ The Fistula Risk Score is a useful tool that can be employed when deciding in which patients to selectively leave a prophylactic surgical drain. Originally develop by Callery et al. ${ }^{9}$ and subsequently validated in a multi-institutional study, the score is comprised of gland texture, pathology, pancreatic duct diameter, and intraoperative blood loss to give a score on a scale of $0-10$ and divides patients into negligible, low, intermediate, and high risk for POPF. ${ }^{9,10}$ Decisions regarding leaving drains, and also managing them, remain extremely variable amongst surgeons. ${ }^{2,8,11,12}$

Kawai and collogues studied 104 patients following

Received: September 6, 2020; Revised: December 7, 2020; Accepted: December 31, 2020

Corresponding author: Rachel E. Beard

Department of Surgery, Rhode Island Hospital, The Lifespan Cancer Institute, Warren Alpert Medical School of Brown University, 2 Dudley Street, Suite 370 Providence, RI 02905, USA

Tel: +1-401-553-8338, Fax: +1-401-868-2302, E-mail: rachel.beard@brownphysicians.org

Copyright (C) 2021 by The Korean Association of Hepato-Biliary-Pancreatic Surgery

This is an Open Access article distributed under the terms of the Creative Commons Attribution Non-Commercial License (http://creativecommons.org/ licenses/by-nc/4.0) which permits unrestricted non-commercial use, distribution, and reproduction in any medium, provided the original work is properly cited. Annals of Hepato-Biliary-Pancreatic Surgery • pISSN: 2508-5778 • elSSN: 2508-5859 
pancreaticoduodectomy and found that intra-abdominal infections were independently associated with patients prospectively assigned to have drains left in place for a longer period of time ( 8 days compared with 4 days). ${ }^{13}$ Based on these data and similar reports, efforts have turned to early drain removal when a drain is left in place, with a variety of groups reporting various drain amylase values predictive of safe drain removal at post-operative day (POD) 1 or $3 .^{2,7,8,11}$ While useful, drain amylase lacks specificity for clinically relevant POPF and is limited in identifying POPF only in patients with surgical drains in place. As POPF can lead to infection, sepsis, and septic shock, the aim of this study is to investigate the known immunologic alternations in response to sepsis, specifically lymphopenia, to identify an additional early readily available marker to improve specificity for only clinically relevant POPF. ${ }^{14,15}$ Lymphopenia has previously been demonstrated to be associated with increased mortality secondary to sepsis and trauma. ${ }^{16,17}$ Sepsis is purported to drive lymphopenia by inducing lymphocyte apoptosis to prevent an overly exuberant inflammatory response to bacterial infection. ${ }^{18-20}$ Thus, unlike drain amylase, lymphopenia has the potential to be more specific for clinically relevant POPF with the added utility of recognizing POPF in patients without prophylactic intraperitoneal drains.

\section{MATERIALS AND METHODS}

All patients who underwent pancreaticoduodenectomy in a single hospital system between July 2008 and June 2018 were identified. The medical records of these patients were retrospectively reviewed to create a comprehensive database including patient demographics, comorbidities, functional status, operative details, post-operative course, pathologic data, and basic laboratory values. All patients over the age of 18 with available operative reports and discharge summaries were included in the analysis. Patients were followed for a minimum of 90 days or until death. Pre-operative lab values were included when drawn within the two weeks prior to pancreaticoduodenectomy. Patients were excluded from analysis if they did not have complete blood counts with differentials available on POD \#3. POD \#3 was selected given prior reports in which drain amylase was used as an indicator of safety for drain removal at this time point ${ }^{2,8}$

POPF were defined using the 2016 update of the ISGPS definition and grading of postoperative pancreatic fistula as described elsewhere. ${ }^{1}$ Briefly, all patients had a drain amylase $>3$ times the upper limit of normal for serum with a clinically relevant alteration of the post-operative course. Biochemical leaks without a deviation from the normal post-operative course are not considered POPF. Grade B POPF indicates persistent drainage for $>3$ weeks, signs of infection, or a percutaneous, endoscopic, or angiographic procedure. Grade C POPF indicates organ failure, need for re-operation, or death. Patients were considered to have POPF only if they met the ISGPS criteria for grade B and C. Operative complications were stratified by the Clavien-Dindo classification system; all ClavienDindo class 2 through 5 were characterized as requiring intervention for their complications. ${ }^{21}$ Patient's pre-operative comorbidities were scored using the Charlson-Deyo Index.

Lymphocyte counts were extracted from the medical record from standard complete blood counts (CBC) with differentials. Leukocytosis and lymphocyte count were defined based on the clinical laboratory standard at Rhode Island Hospital with normal ranges of 4,000-11,000 $\mu \mathrm{l}$ and $1,000-4,000 / \mu 1$ respectively. Patients were defined as having persistent lymphopenia if the absolute lymphocyte count failed to normalize by POD \#3.

$\mathrm{R}$ version 5.3.1 (The R Foundation for Statically Computing) was used for data analysis. A $p$-value of 0.05 was employed to define significance for all tests when reported. Continuous variables were analyzed by Mann Whitney U test and expressed as medians and ranges. Categorical variables were analyzed by $X^{2}$ test and expressed as absolute numbers and percentages. Univariate Cox proportional hazard regression was performed for patient, operative, and clinicopathologic data using POPF as the outcome. Initially multivariate Cox proportional hazard regression was then performed including all factors from the univariate analysis. A backwards stepwise elimination of all non-significant variables was subsequently performed until all remaining variables were significantly associated with POPF. This study was approved by the Rhode Island Hospital institutional review board, informed consent was waved for this retrospective review. 


\section{RESULTS}

201 patients underwent pancreaticoduodenectomy in the study period. 40 patients were excluded due to lack of lymphocyte counts. Of the remaining patients, 81 had lymphopenia and 80 had normal lymphocyte counts on POD \#3. There were 17 POPF in patients with persistent lymphopenia which was significantly greater when compared with the 7 POPF in patients with normal lymphocyte counts on POD \#3 ( $p=0.032)$. Twenty-four patients (14.9\%) developed ISGPS Grade B and C POPF. Persistent lymphopenia had a sensitivity of $70.8 \%$ and a specificity of $53.3 \%$, as well a negative predictive value of $91.3 \%$ and a positive predictive value of $21.0 \%$ for POPF (Fig. 1).

The median patient age was 67 years old with a range from 23 years old to 92 years old. The majority of patients $(88 / 161,54.7 \%)$ in this study were male, and patients with persistent lymphopenia were significant more likely to be male $(p=0.033)$. Patients with POD \#3 lymphopenia were significantly more likely to be lymphopenic pre-operative ( $2 \%$ vs $17.2 \%, p=0.009)$. Just over half of patients $(50.3 \%)$ demonstrated leukocytosis at POD \#3. There was no significant difference in the proportion of patients with POD \#3 leukocytosis between patients with and without persistent lymphopenia. Further, there was no significant difference in the frequency with which patients received neo-adjuvant chemotherapy, nor was there a difference in the frequency with which patients underwent biliary drainage or stenting procedures. By Charlson-Deyo score the two cohorts had similar severity of pre-operative comorbidities (Table 1).

The most common indication for pancreaticoduodenectomy was pancreatic adenocarcinoma (95/161 patients, $59.0 \%$ ), followed by peri-ampullary or ampullary adenocarcinoma (28/161 patients, $17.4 \%$ ), and intraductal papillary mucosal neoplasms (14/161 patients, 8.7\%). Patients with and without persistent lymphopenia had similar patterns of tumor types. Sixteen of the 161 patients (10\%) underwent vascular resection at the time of their operation and 22 patients $(13.7 \%)$ required intraoperative transfusion. All patients in the study had prophylactic intra-peritoneal drains lefts at the time of their index operation.

There were no significant differences in overall survival (OS) on Kaplan-Meier analysis with a median OS of 93.9 months (IQR 21.7 months-upper limit undefined) in patients without persistent lymphopenia compared with 45.9 (IQR 17.6 months to 120.8 months) in patients with persistent lymphopenia $(p=0.17)$ (Fig. 2A). When patients were subdivided into those with persistent lymphopenia, those that were initially lymphopenic but had count recovery by POD\# 3, and those that were never lymphopenic, there was similarly no significant difference in median OS at 49.5 months (IQR 15.0 months-upper limit undefined), 55.6 months (IQR 13.2 months-upper limit undefined), and 93.9 (IQR 7.9 months-upper limit undefined) re-

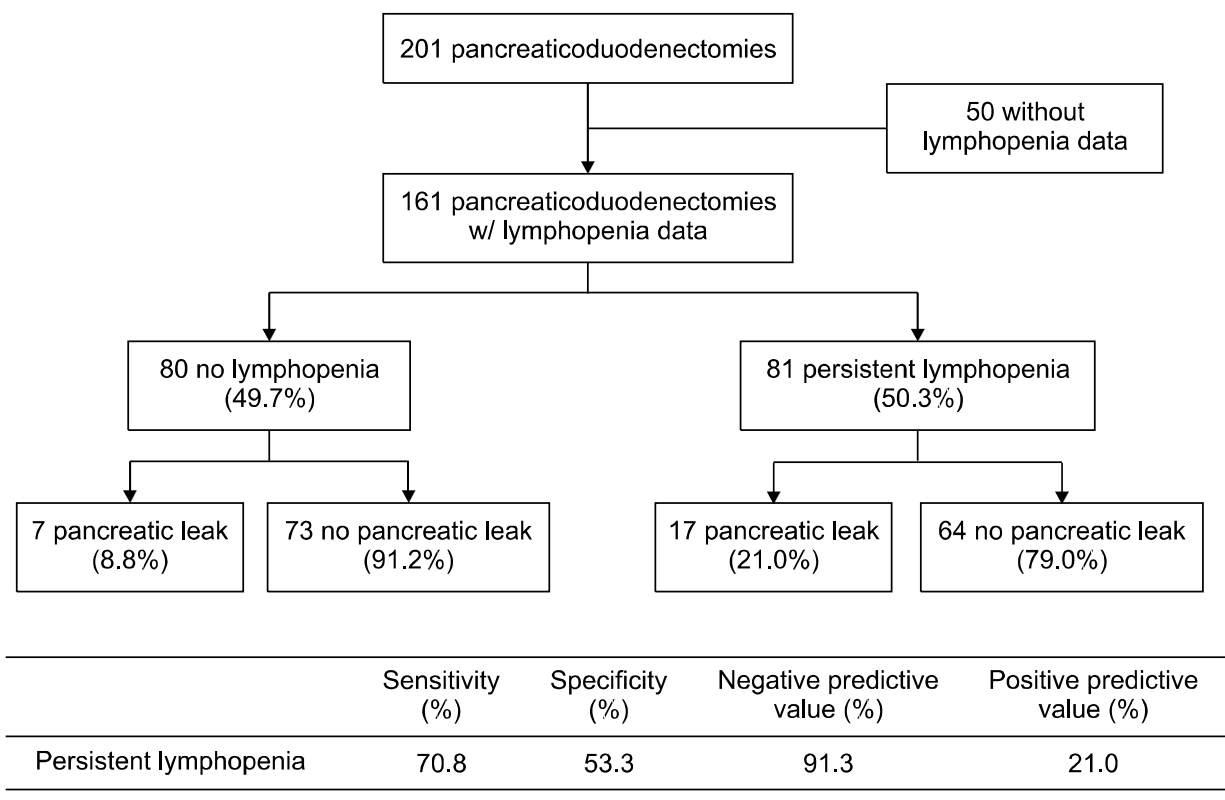

Fig. 1. Flow chart of pancreatic leak patterns with sensitivity, specificity, positive predictive value and negative predicted value calculated. 
Table 1. Clinical characteristics by lymphopenia

\begin{tabular}{|c|c|c|c|}
\hline Variable & $\begin{array}{c}\text { No lymphopenia } \\
\mathrm{n}=80(\%)\end{array}$ & $\begin{array}{c}\text { Persistent lymphopenia } \\
n=81(\%)\end{array}$ & $p$ value \\
\hline Age, median (years) [range] & $65[25-92]$ & $67[23-85]$ & 0.156 \\
\hline Male sex & $37(46.3)$ & $51(63.0)$ & 0.033 \\
\hline Charlson-Deyo Score, median [range] & $4[0-9]$ & $4[0-9]$ & 0.753 \\
\hline Pre-operative lymphopenia & $1(2)$ & $10(17.2)$ & 0.009 \\
\hline POD\#3 leukocytosis & $43(53.8)$ & $38(46.9)$ & 0.386 \\
\hline Neo-adjuvant therapy & $4(5)$ & $5(6.2)$ & 0.746 \\
\hline Pre-operative stenting/drainage procedure & $23(28.75)$ & $22(27.2)$ & 0.822 \\
\hline Tumor type & & & 0.122 \\
\hline Pancreatic adenocarcinoma & $54(66.7)$ & $41(50.6)$ & \\
\hline Peri-ampullary/ampullary adenocarcinoma & $13(16.3)$ & $15(18.5)$ & \\
\hline IPMN & $3(3.75)$ & $11(13.6)$ & \\
\hline Inflammatory & $2(2.5)$ & $2(2.5)$ & \\
\hline Other & $8(10)$ & $12(14.8)$ & \\
\hline Vascular resection & $7(8.8)$ & $9(11.1)$ & 0.617 \\
\hline Lymphovascular invasion & $37(46.3)$ & $41(50.6)$ & 0.579 \\
\hline Perineural invasion & $48(60)$ & $52(64.2)$ & 0.583 \\
\hline Intraoperative Transfusion & $8(10.7)$ & $14(18.4)$ & 0.177 \\
\hline Pancreatic duct diameter $(\mathrm{mm})$, median [range] & $5[1.5-10]$ & $4[2-15]$ & 0.594 \\
\hline Gland texture & & & 0.837 \\
\hline Soft & $1(50)$ & $3(33.3)$ & \\
\hline Intermediate & $0(0)$ & $1(11.1)$ & \\
\hline Firm & $1(50)$ & $5(55.6)$ & \\
\hline
\end{tabular}

\section{A}

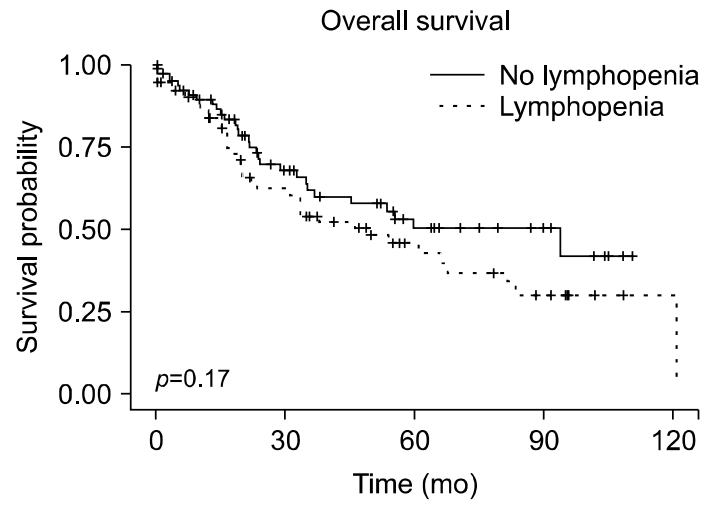

Number at risk

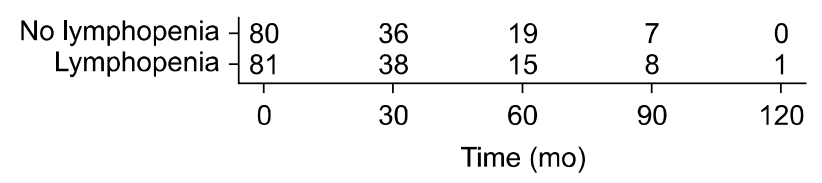

Fig. 2. Kaplan Meier analysis of (A) overall survival and by lymphopenia status.

spectively $(p=0.28)$. Looking specifically at patients with pancreatic ductal adenocarcinoma, there was no significant difference in progression free survival (PFS) with a median PFS of 18.9 months (IQR 11.4 months-81.6 months) without persistent lymphopenia compared with 15.4 months (9.8 months-39.7 months) with persistent lymphopenia
B

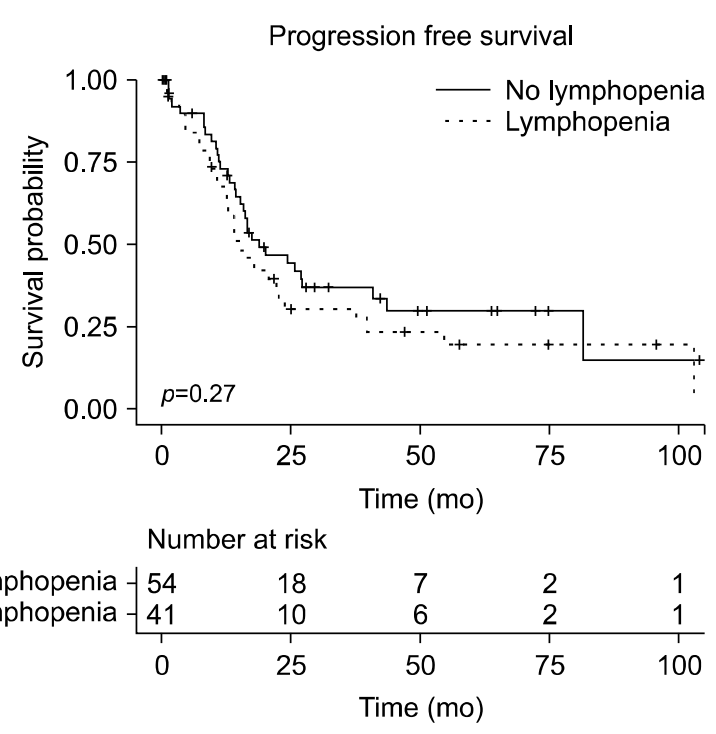

(B) Progression free survival of pancreatic ductal adenocarcinoma

$(p=0.27)$ (Fig. 2B).

Using POPF as the outcome, univariate analysis was performed on a variety of patient, disease, and operative characteristics. Only POD\#3 lymphopenia was significantly associated with POPF. Subsequently multivariate analysis with POPF as the outcome was performed with all var- 
iables from the univariate analysis employing a backwards stepwise elimination model. Following backward elimination, only POD \#3 lymphopenia was independently associated with POPF (HR 2.57, 95\% CI 1.07-6.64, $p=0.039$ ) (Table 2). There was no independent association of neoadjuvant therapy, preoperative lymphopenia, or POD \#3 leukocytosis with POPF. Within the known predictors of POPF, there was no association with intraoperative blood loss or tumor type. There were not enough data points for gland texture or pancreatic duct diameter to be included in the univariate and multivariate analysis.

Overall, 54 of the 161 patients (33.5\%) had no complications following their index operation. Another 33 patients $(20.5 \%)$ had a minor complication that did not require major intervention (Clavien-Dindo class 1). Between patients with and without persistent lymphopenia, there was no significant difference in the number of patients without complications or a complication not requiring an intervention. Patients with persistent lymphopenia were significantly more likely to have a complication that required intervention $(p<0.001)$ (Table 3$)$. The majority of complications requiring intervention were Clavien-Dindo class 2 (40/74 complications, 52.6\%). In total, there were nine patients that suffered organ failure $(5.6 \%)$ and six peri-operative deaths $(3.7 \%)$. Of those patients that died, three were the result of hemorrhage, two were secondary to septic complications, and one was due to a pulmonary embolism. Perioperative deaths were not significantly dif- ferent between patients with persistent lymphopenia compared to those without at five and one deaths respectively $(p=0.099)$.

There were significant differences in the number of patients that required a variety of interventions for their complications when comparing patients with and without persistent lymphopenia. Overall 42 patients (26.1\%) required antibiotics to treat infection, with twice as many patients with persistent lymphopenia requiring antibiotics when compared to patients without persistent lymphopenia (28 patients vs 14 patients), which was statistically significant $(p=0.014)$. Twelve total patients required reoperation for the management of a complication, including eight patients with septic complications and four patients with hemorrhagic complications. Patients with persistent lymphopenia were significantly more likely to require re-operation (10 patients) compared to patients without persistent lymphopenia ( 2 patients) $(p=0.017)$. Overall the median length of stay was 9 days, and patients with persistent lymphopenia had a one day longer median length of stay compared to patients without persistent lymphopenia, which was statistically significant $(p=0.013)$. The number of patients with surgical drains present at discharge, that required percutaneous drainage, and who were re-admitted within 30-days was similar between the two cohorts (Table 3).

Table 2. Univariate and multivariate analysis of clinical variables in relation to pancreatic leaks

\begin{tabular}{lccc}
\hline \multicolumn{1}{c}{ Variable } & & Univariate & Multivariate \\
\cline { 2 - 3 } & & $p$ value Hazard ration (CI) & $p$ value \\
\hline Age $\geq 75$ & 44 & 0.14 \\
Male sex & 88 & 0.38 \\
Vascular resection & 16 & 0.31 \\
Intraoperative transfusion & 22 & 0.45 \\
EBL >400 & 73 & 0.63 \\
Multiorgan resection & 15 & 0.31 \\
History of diabetes & 50 & 0.84 \\
Pre-operative stenting & 61 & 0.95 \\
Neo-adjuvant therapy & 9 & 0.99 \\
Pancreatic adenocarcinoma/chronic inflammation & 99 & 0.66 \\
Preoperative lymphopenia & 11 & 0.094 \\
POD\#3 leukocytosis & 81 & 0.746 \\
POD\#3 lymphopenia & 81 & 0.039 \\
\hline
\end{tabular}

CI, confidence interval; EBL, estimated blood loss; POD, post-operative day 
Table 3. (A) Comparison of complication by Clavien-Dindo classification between normal and persistently lymphopenic patients. (B) Comparison of adverse events between normal and persistently lymphopenic patients

\begin{tabular}{|c|c|c|c|c|}
\hline & Overall & $\begin{array}{c}\text { No lymphopenia } \\
n=80(\%)\end{array}$ & $\begin{array}{c}\text { Persistent } \\
\text { lymphopenia } \\
n=81 \quad(\%)\end{array}$ & $p$ value \\
\hline \multicolumn{5}{|l|}{ (A) Complication } \\
\hline None & 54 & $31(38.8)$ & $23(28.4)$ & 0.160 \\
\hline \multicolumn{5}{|l|}{ No intervention required } \\
\hline Class 1 & 33 & $21(26.3)$ & $12(14.8)$ & 0.078 \\
\hline Intervention required & & & & $<0.001$ \\
\hline Class 2 & 40 & $16(20)$ & $24(29.6)$ & \\
\hline Class 3 & 19 & $9(11.3)$ & $10(12.3)$ & \\
\hline Class 4 & 9 & $2(2.5)$ & $7(8.6)$ & \\
\hline Class 5 & 6 & $1(1.3)$ & $5(6.2)$ & \\
\hline \multicolumn{5}{|l|}{ (B) Management of complications } \\
\hline Antibiotics & 42 & $14(17.5)$ & $28(34.6)$ & 0.014 \\
\hline $\begin{array}{l}\text { Time to removal of surgical drain, } \\
\text { median (days) [range] }\end{array}$ & 6 & $5[3-29]$ & $6[3-28]$ & 0.942 \\
\hline Surgical drain present at discharge & 108 & $52(65.0)$ & $56(69.1)$ & 0.576 \\
\hline Percutaneous drainage & 23 & $10(12.5)$ & $13(16.0)$ & 0.520 \\
\hline Re-operation & 12 & $2(2.5)$ & $10(12.3)$ & 0.017 \\
\hline Hospital LOS, median (days) [range] & 9 & $8[2-42]$ & $9[5-133]$ & 0.013 \\
\hline ICU LOS, median (days) [range] & 0 & $0[0-11]$ & $0[0-120]$ & 0.126 \\
\hline 30-day readmission & 35 & $15(18.8)$ & $20(24.7)$ & 0.361 \\
\hline
\end{tabular}

LOS, length of stay; ICU, intensive care unit

\section{DISCUSSION}

Given the variable practice patterns, frequency of POPF, and consequences of unrecognized POPF, there exists a need for early readily available markers. Here we demonstrate that persistent lymphopenia on POD \#3 is independently associated with clinically relevant POPF. Perhaps, more importantly, we note that a normal lymphocyte count on POD \#3 had over 91\% negative predictive value for POPF. This result is likely due to a degree of immunosuppression, which has been well described following trauma, major operations, and the onset of sepsis. ${ }^{14,15,20,22}$

In response to major surgery, lymphocyte apoptosis increases which can subsequently decrease the absolute lymphocyte count for the first 72-96 hours post-operatively. ${ }^{16,20,23}$ Sepsis similarly leads to increase in CD-4 helper T-Cell and B-cell apoptosis in a time dependent progressive manner. That is, the longer sepsis is present the more profound the degree of apoptosis becomes. ${ }^{18}$ When sepsis is superimposed on the initial insult of an operation, prolonged lymphopenia results with failure to replenish circulating lymphocytes. This persistent immunosuppression has been linked to late mortality from sepsis. ${ }^{17,22}$
Even in the absence of sepsis, persistent lymphopenia has been associated with increased mortality at three weeks following a traumatic injury. Interestingly, here patients with early lymphopenia that recovered by post-injury day 4 had similar survival to patients that were never lymphopenic; however, when analyzing patients that did die, this cohort had the longest time to death. This suggests transient immune cell exhaustion and desensitization is likely protective, but with ongoing insults a persistent lymphopenic response becomes detrimental. ${ }^{16}$

In the present study, patients were not significantly more likely to die in the perioperative period with persistent lymphopenia when compared to patients with normal lymphocyte counts on POD \#3, and overall survival was not significantly different between the two groups. However, this may be due in part to the low event rate given there were only 6 total perioperative deaths. Indeed, patients with persistent lymphopenia accounted for 5 of the 6 total perioperative deaths and had half as long median OS. Further, OS is likely clouded by underlying tumor biology and indication for pancreaticoduodenectomy.

These data run counter to reports of utilizing pre-operative neutrophil-to-lymphocyte ratios in the prediction of 
overall- and disease free-survival following pancreaticoduodenectomy for pancreatic adenocarcinoma. ${ }^{24}$ However, these studies examine pre-operative cell counts which reflect the immunomodulatory effects of the tumor itself rather than the post-operative counts which more likely reflect the pathologic insult of surgery and ongoing inflammation from POPFs.

In patients with POPF, the ongoing enzymatic leak and superimposed inflammation and infection would act as a continuous insult leading to lymphocyte exhaustion and apoptosis. Given the limited role of lymphocytes in the first five days of wound healing it is unlikely that lymphopenia would impact anastomotic integrity. ${ }^{25}$ Thus persistent lymphopenia is more likely to act as a marker of impending sepsis secondary to a POPF rather than a causative factor contributing to the development of a POPF. An assertion that is supported by the 2-fold increase in the need for antibiotics, a 5-fold increase in the need for re-operation observed in this study.

Patients with lymphopenia were nearly 2.5 times more likely to develop a POPF when compared to patients without persistent lymphopenia. This finding is independent of pre-operative lymphopenia as well as leukocytosis at the same time point. Patients with persistent lymphopenia had a $170 \%$ increase in the rate of complications that required intervention and a one day increase in the median length of hospitalization. We were unable to stratify these patients based on their Fistula Risk Score, as duct diameter and gland texture were not routinely dictated in operative notes, which is a limitation of this study.

Although all of the patients included here had prophylactic intraperitoneal drains left at the time of their pancreaticoduodenectomy, extrapolating from these data there may be a readily available early marker for the development of POPF in patient where no drain was left and thus no drain amylase is available for testing. Using persistent lymphopenia as a marker for POPF has a negative predictive value of just over $91 \%$, which is a useful data point in the evaluation of an often complex post-pancreaticoduodenctomy patient deviating from the normal post-operative course, especially when no drain amylase is available. In addition to the pancreatic Fistula Risk Score, there was recently a nomogram for predicting clinically relevant POPF. $^{26}$ As these tools are refined and become available, it is likely that selective draining will be- come more commonplace and the early recognition of POPF will be critical to prevent delays in management. In these patients, persistent lymphopenia could prompt early diagnostic imaging studies allowing for early percutaneous or endoscopic interventions, ultimately preventing the need for emergent re-operative interventions. Further, identifying a patient cohort at risk of developing clinically significant POPF could avoid inappropriately early discharges and prevent the readmission of patients in extremis. Unfortunately, in the current study, duct diameter and gland texture were only available in a small number of cases, so we were unable to stratify the negative predictive value of lymphopenia by Fistula Risk Score.

Further, while early drain amylase is promising, it is limited in the number of patients that can be identified for early drain removal. For example, Seykora et al. ${ }^{11}$ proposed a drain amylase cut-off of $\leq 150$ IU/L for drain removal on POD\#3 for a negative predictive value of $96.6 \%$. However, at this cut-off there is only a $36.6 \%$ specificity, which leaves a large cohort of patients with drains in place that will not go onto develop POPF. Serene et al. ${ }^{2}$ improved specificity to $56 \%$ by combining POD \#1 and POD \#3 drain amylase, which is similar to the specificity we report here. As early drain removal becomes prevalent, persistent lymphopenia may become an important indicator of POPF after drain removal when drain amylase is no longer available. Additionally, combining persistent lymphopenia with drain amylase may allow for early removal of surgical drains at a higher drain amylase threshold by improving specificity for clinically relevant POPF, although prospective validation is required.

Drain amylase was not routinely checked on POD \#3 at our institution during the study period, though it has since been adopted. We are therefore unable to comment on the sensitivity and specificity of combining drain amylase and persistent lymphopenia beyond that a combination holds the potential for improved prognostic capability. This study is further limited as drain management was not standardized and was left to the discretion of the five operating surgeons contributing to the study. Additional studies would be required but combining persistent lymphopenia with drain amylase levels on POD \#3 has the potential to liberalize the drain amylase cut-off improving specificity and positive predictive value while maintaining the negative predictive value. 
In conclusion persistent lymphopenia following pancreaticoduodenectomy is independently associated with POPF likely owing to the immunosuppressive effects of impending sepsis. As such, persistent lymphopenia can act as an adjunct to drain amylase in the early detection and management of clinically relevant POPF when an intraperitoneal drain is present. Further, persistent lymphopenia holds the potential to act as a readily available early marker of developing POPF in patients where no intraperitoneal drain is present. Prospective validation in patients and with and without intraperitoneal drains is an important next step in fully elucidating the potential of persistent lymphopenia as a predictor of POPF.

\section{ORCID}

Joshua T. Cohen: https://orcid.org/0000-0002-6552-6578

Kevin P. Charpentier: https://orcid.org/0000-0002-9561-1503

Thomas J. Miner: https://orcid.org/0000-0002-0215-7314

William G. Cioffi: https://orcid.org/0000-0001-8192-8836

Rachel E. Beard: https://orcid.org/0000-0002-2781-2151

\section{AUTHOR CONTRIBUTIONS}

Conceptualization: JTC, KPC, TJM, WGC, REB. Data curation: JTC, KPC, TJM. Formal analysis: JTC, REB. Funding acquisition: N/A. Methodology: JTC, WGC, REB. Project administration: JTC, REB. Visualization: JTC. Writing original draft: JTC, TJM, REB. Writing - review \& editing: JTC.

\section{REFERENCES}

1. Bassi C, Marchegiani G, Dervenis C, Sarr M, Abu Hilal M, Adham M, et al. The 2016 update of the International Study Group (ISGPS) definition and grading of postoperative pancreatic fistula: 11 years after. Surgery 2017;161:584-591.

2. Serene TEL, G SV, Padmakumar JS, Terence HCW, Keem LJ, Bei W, et al. Predictive value of post-operative drain amylase levels for post-operative pancreatic fistula. Ann Hepatobiliary Pancreat Surg 2018;22:397-404.

3. Pedrazzoli S. Pancreatoduodenectomy (PD) and postoperative pancreatic fistula (POPF): a systematic review and analysis of the POPF-related mortality rate in 60,739 patients retrieved from the English literature published between 1990 and 2015. Medicine (Baltimore) 2017;96:e6858.

4. Schäfer M, Müllhaupt B, Clavien PA. Evidence-based pancreatic head resection for pancreatic cancer and chronic pancreatitis. Ann Surg 2002;236:137-148.
5. Van Buren G 2nd, Bloomston M, Hughes SJ, Winter J, Behrman $\mathrm{SW}$, Zyromski NJ, et al. A randomized prospective multicenter trial of pancreaticoduodenectomy with and without routine intraperitoneal drainage. Ann Surg 2014;259:605-612.

6. Witzigmann H, Diener MK, Kienkötter S, Rossion I, Bruckner $\mathrm{T}$, Werner B, et al. No need for routine drainage after pancreatic head resection: the dual-center, randomized, controlled PANDRA trial (ISRCTN04937707). Ann Surg 2016;264:528-537.

7. McMillan MT, Malleo G, Bassi C, Butturini G, Salvia R, Roses $\mathrm{RE}$, et al. Drain management after pancreatoduodenectomy: reappraisal of a prospective randomized trial using risk stratification. J Am Coll Surg 2015;221:798-809.

8. Lee SR, Kim HO, Shin JH. Significance of drain fluid amylase check on day 3 after pancreatectomy. ANZ J Surg 2019;89:497502.

9. Callery MP, Pratt WB, Kent TS, Chaikof EL, Vollmer CM Jr. A prospectively validated clinical risk score accurately predicts pancreatic fistula after pancreatoduodenectomy. J Am Coll Surg 2013;216:1-14.

10. Miller BC, Christein JD, Behrman SW, Drebin JA, Pratt WB, Callery MP, et al. A multi-institutional external validation of the fistula risk score for pancreatoduodenectomy. J Gastrointest Surg 2014;18:172-179; discussion 179-180.

11. Seykora TF, Maggino L, Malleo G, Lee MK 4th, Roses R, Salvia $R$, et al. Evolving the paradigm of early drain removal following pancreatoduodenectomy. J Gastrointest Surg 2019;23: 135-144.

12. Wang Q, Jiang YJ, Li J, Yang F, Di Y, Yao L, et al. Is routine drainage necessary after pancreaticoduodenectomy? World J Gastroenterol 2014;20:8110-8118.

13. Kawai M, Tani M, Terasawa $H$, Ina $S$, Hirono $S$, Nishioka $R$, et al. Early removal of prophylactic drains reduces the risk of intra-abdominal infections in patients with pancreatic head resection: prospective study for 104 consecutive patients. Ann Surg 2006;244:1-7.

14. Venet F, Davin F, Guignant C, Larue A, Cazalis MA, Darbon $\mathrm{R}$, et al. Early assessment of leukocyte alterations at diagnosis of septic shock. Shock 2010;34:358-363.

15. Munford RS, Pugin J. Normal responses to injury prevent systemic inflammation and can be immunosuppressive. Am J Respir Crit Care Med 2001;163:316-321.

16. Heffernan DS, Monaghan SF, Thakkar RK, Machan JT, Cioffi WG, Ayala A. Failure to normalize lymphopenia following trauma is associated with increased mortality, independent of the leukocytosis pattern. Crit Care 2012;16:R12.

17. Drewry AM, Samra N, Skrupky LP, Fuller BM, Compton SM, Hotchkiss RS. Persistent lymphopenia after diagnosis of sepsis predicts mortality. Shock 2014;42:383-391.

18. Hotchkiss RS, Tinsley KW, Swanson PE, Schmieg RE Jr, Hui JJ, Chang KC, et al. Sepsis-induced apoptosis causes progressive profound depletion of B and CD4+ T lymphocytes in humans. J Immunol 2001;166:6952-6963.

19. Le Tulzo Y, Pangault C, Gacouin A, Guilloux V, Tribut O, Amiot L, et al. Early circulating lymphocyte apoptosis in human septic shock is associated with poor outcome. Shock 2002;18: 487-494.

20. Girardot T, Rimmelé T, Venet F, Monneret G. Apoptosis-induced lymphopenia in sepsis and other severe injuries. Apoptosis 2017;22:295-305.

21. Dindo D, Demartines N, Clavien PA. Classification of surgical complications: a new proposal with evaluation in a cohort of 6336 patients and results of a survey. Ann Surg 2004;240:205213.

22. Venet F, Rimmelé T, Monneret G. Management of sepsis-in- 
duced immunosuppression. Crit Care Clin 2018;34:97-106.

23. Hotchkiss RS, Karl IE. The pathophysiology and treatment of sepsis. N Engl J Med 2003;348:138-150.

24. Templeton AJ, McNamara MG, Šeruga B, Vera-Badillo FE, Aneja P, Ocaña A, et al. Prognostic role of neutrophil-to-lymphocyte ratio in solid tumors: a systematic review and meta-analysis.
J Natl Cancer Inst 2014;106:dju124.

25. Park JE, Barbul A. Understanding the role of immune regulation in wound healing. Am J Surg 2004;187:11S-16S.

26. You Y, Han IW, Choi DW, Heo JS, Ryu Y, Park DJ, et al. Nomogram for predicting postoperative pancreatic fistula. HPB (Oxford) 2019;21:1436-1445. 